\title{
Estrés parental, trato rudo y monitoreo como factores asociados a la conducta agresiva*
}

Parental stress, harsh treatment and parental monitoring as factors associated with aggressive behavior

Recibido: agosto 2 de 2010 | Revisado: octubre 20 de 2010 | Aceptado: noviembre 8 de 2010

\author{
Victoria EugEnia CABrera García ** \\ MARTHA Rocío GONZÁlez BERNAL \\ Facultad de Psicología, \\ Universidad de La Sabana, Bogotá, Colombia \\ Ivón PaOla Guevara Marín **** \\ Instituto de La Familia, \\ Universidad de La Sabana, Bogotá, Colombia
}

SICI: 2011-2777(201203)11:1<241:EPTRYM>2.0.CO;2-Y

Para citar este artículo: Cabrera, V. E., González, M. R. \& Guevara, I. P. (2012). Estrés parental, trato rudo y monitoreo como factores asociados a la conducta agresiva. Universitas Psychologica, 11(1), 241-254.

* Articulo producto del proyecto de investigación "Relaciones maritales, relaciones parentales y su influencia en el ajuste psicológico de los hijos", financiado por la Universidad de La Sabana, realizado en el año 2006 y registrado con código FAM5-2006.

** Facultad de Psicología, Campus del Puente del Común, Km. 7, Autopista Norte de Bogotá, Chía, Cundinamarca, Colombia. E-mail: martha.gonzalez@unisabana.edu.co,victoria.cabrera@unisabana.edu.co.

*** Instituto de La Familia, Campus del Puente del Común, Km. 7, Autopista Norte de Bogotá, Chía, Cundinamarca, Colombia.E-mail: ivon.guevara@ unisabana.edu.co

\section{RESUMEN}

Esta investigación examinó el aporte de tres prácticas parentales en la explicación de la conducta agresiva. El interés era indagar asociaciones entre estos factores, las diferencias por nivel socioeconómico y las diferencias en los reportes de ambos padres sobre la conducta agresiva de sus hijos. Participaron 256 parejas, con hijos adolescentes. Los resultados muestran que el estrés parental, el trato rudo y el monitoreo se asocian significativamente con la conducta agresiva. El estrés parental fue el factor con mayor peso predictor. Se encontraron diferencias significativas para los tres factores en los niveles socioeconómicos alto y bajo, pero no entre el medio y el alto. En los reportes de los padres, no se encontraron diferencias para estrés y trato rudo, pero sí para el monitoreo.

\section{Palabras clave autores:}

Conducta agresiva, factores de riesgo, factores protectores y prácticas parentales.

Palabras clave descriptores:

Índice de Estrés Parental-Forma Corta (PSI/SF), Estudio descriptivo-correlacional.

\section{A B S T R A C T}

This research studied the joint contribution of three parenting practices in the explanation of aggressive behavior. The main interest was to investigate the associations between these factors, the socioeconomic status, and the differences between the reports provided by parents in regards to the aggressive behavior of their children. The sample included 256 couples whose children were teenagers with an age range between 12 and 18 years old. The results show that parental stress, the harsh treatment, and monitoring are significantly associated with aggressive behavior of children. Parental stress was the factor with the highest degree of prediction. Significant differences were found for the three factors in high and low socioeconomic levels, but in medium and high were not. As for the versions of the parents, there were no significant differences in stress and rough management, but monitoring. Key words authors:

Aggressive behavior, risk and protective factors and parental practices.

Key words plus:

Parental Stress Index-Short Form (PSI/SF), descriptive-correlational study 


\section{Introducción}

La agresión entre niños en diferentes escenarios sociales se ha convertido en un fenómeno de relevancia para los investigadores en todo el mundo y puede llegar a considerarse hoy en día como un problema social (Crick \& Pepler, 2007; Guevara, Cabrera \& Barrera, 2007; Morales, García de la Cadena \& Grazioso, 2006; Olweus, 1993; Salmivalli, Lagerspetz, Björkqvist, Österman \& Kaukiainen, 1996). Esta agresión se manifiesta de diversas formas en distintas edades y tiene consecuencias negativas a largo plazo (Crick, Casas \& Mosher, 1997; Gladstone, Parker \& Malhi, 2006; Olweus, 1993). En términos de Cavell (2000), la generalización del comportamiento agresivo parece ser el antecesor de la progresión hacia el comportamiento antisocial hasta llegar a la edad adulta.

En la aparición de la conducta agresiva en niños ${ }^{1}$ y adolescentes parecen influir diferentes factores y, a partir de una postura teórica ecológica, se propone una mirada multidimensional de la conducta agresiva que incluye factores familiares, escolares, personales, socioeconómicos, entre otros (Dekovic, Wissink \& Meijer, 2004).

En el caso del factor familiar que interesa a esta investigación, se encuentra que la familia tiene una particular relevancia en la prevención y generación de la conducta agresiva en los hijos (Ayala, Pedroza, Morales, Chaparro \& Barragán, 2002; Peligrín \& Garcés de Los Fayos, 2008; Richaud de Minzi, 2002). Así, las relaciones padres-hijos pueden favorecer las interacciones familiares, fortalecer los vínculos y crear un clima familiar que contribuya al desarrollo humano, pero también pueden presentarse relaciones padres-hijos que son estresantes y en las cuales los padres perciben que tener hijos y la relación con ellos es una carga. Esta forma de relaciones puede generar trato rudo de los padres hacia los hijos y sentimientos de malestar de los hijos hacia los padres, ocasionando en ellos conductas desadaptativas como la agresión (Burke \& Abidin,

1 Los términos niños e hijos se refieren a niños y niñas, hijos e hijas, respectivamente.
1980; Pinderhughes, Dodge, Bates, Pettit \& Zelli, 2000; Raya, Pino \& Herruzo, 2009).

Por el contrario, padres que utilizan prácticas paternas como el monitoreo o la supervisión favorecen el desarrollo de comportamientos sociales adaptativos, convirtiéndose en factores protectores frente a la generación de conductas agresivas (Raya et al., 2009).

Este estudio se centra en tres factores familiares asociados a la conducta agresiva, pero que en la literatura no han sido estudiados en conjunto. Estos factores son: el estrés parental y el trato rudo como factores de riesgo y el monitoreo como factor protector. Así, el propósito del estudio es describir la interacción de estos tres factores y su contribución en la aparición de conductas agresivas en los hijos.

\section{Conducta agresiva}

La conducta agresiva es definida en los trabajos de Achenbach y Rescola (2001) como una conducta externalizante que inicia en la niñez y puede evolucionar en la edad adulta a formas severas como la conducta antisocial (Ayala, Fulgencio, Chaparro \& Pedroza, 2000; Côté, Vaillancourt, Barker, Nagin $\&$ Tremblay, 2007). Stone, Buehler y Barber (2002) explican que se denominan problemas externalizantes a conductas que se reflejan externamente, tales como la agresión, el robo o hurto y la mentira.

La conducta externalizante agresiva consiste en una acción negativa que se produce cuando alguien, de manera intencionada, causa un daño o hiere a otra persona (Olweus, 1993). Esta conducta, de manera general, se expresa en tres tipos: agresión física, agresión verbal y agresión indirecta, relacional o social.

La agresión física se caracteriza por el daño físico que se la hace a otra persona, mientras que la agresión verbal se identifica como una manera de herir a otra persona a través de las palabras (Chaux, 2003; Tremblay, Gervais \& Petitclerc, 2008). La agresión indirecta, relacional o social, incluye acciones como la exclusión, el manejo de rumores y el aislamiento social (Björkqvist, 2001; Galen \& Underwood, 1997; Salmivalli \& Kaukiainen, 2004). 
Considerando la relevancia y prevalencia de las conductas externalizantes, especialmente de la conducta agresiva, los investigadores han hecho especial énfasis en la identificación de factores de riesgo y factores protectores (Peligrín \& Garcés de Los Fayos, 2008).

Según Ayala et al. (2002), los factores de riesgo se han clasificado en cuatro grandes grupos: (a) las características de los niños, (b) las características de los padres, (c) los factores contextuales y (d) las relaciones padre-hijo.

En relación con las características de los niños, se ha encontrado en diferentes estudios que los niños que presentan falta de autocontrol (Farrington, 1989; Peligrín \& Garcés de Los Fayos, 2008), baja tolerancia a la frustración, sobreactividad y dificultades atencionales son más susceptibles de mostrar problemas de conducta que los niños que no presentan estas características (Abidin, 1995; Barkley, 1997; Henao, 2006). Igualmente, son vulnerables a la conducta agresiva aquellos niños que presentan baja consideración y respeto hacia los demás (Dodge, Pettit, McClaskey \& Brown, 1986; Pelegrín, 2004).

En cuanto a las características de los padres, se destaca la forma en que estos perciben el comportamiento de sus hijos. Cuando las percepciones de la conducta de los hijos no son favorables, los niños pueden presentar problemas de ajuste social (Abidin, 1995; Henao, 2006). Estas percepciones a su vez influyen en la interacción que los padres pueden tener con sus hijos. Del mismo modo, el temperamento negativo, la hostilidad, el estrés, la poca cercanía con los hijos y la inmadurez, se relacionan con la conducta agresiva de los niños (Ayala et al., 2000). Este estudio se centró en los factores de riesgo de los padres relativos al estrés parental y el trato rudo hacia los hijos.

Los factores contextuales también se asocian con la conducta agresiva de los niños. Por ejemplo, el aislamiento social de los padres (Peligrín \& Garcés de Los Fayos, 2008; Wahler, 1980), o la falta de recursos de los niveles socioeconómicos más bajos, pueden ejercer presión sobre todos sus miembros y generar conducta agresiva. Igualmente, se destaca la influencia de los medios de comunicación como la televisión y los videojuegos (Peligrín, 2004; Urra, 1998).
El último factor de riesgo tiene que ver con las relaciones padre-hijo como interacción entre las características del niño, las características del padre y los factores contextuales. Cuando se presentan altos niveles de estrés parental, sumado a las dificultades que puede presentar el hijo y a la interacción disfuncional entre padre e hijo, se da un aumento de comportamientos autoritarios y consecuentemente un impacto negativo y directo del estrés sobre el hijo, trayendo como consecuencia el incremento de problemas de ajuste comportamental en los hijos (Peligrín \& Garcés de Los Fayos, 2008).

Por otra parte, los factores protectores son identificados como variables que disminuyen la probabilidad de la presencia de la conducta agresiva externalizante. Kumpfer y Alvarado (1997) clasifican de la siguiente manera los factores protectores que previenen la conducta agresiva: (a) relaciones de apoyo padre e hijo, (b) métodos positivos de disciplina, (c) monitoreo y supervisión, (d) familias dedicadas a sus hijos y (e) padres que buscan información y apoyo.

Algunos autores indican que la supervisión de los padres, su apego a los hijos y la congruencia en la disciplina son los factores protectores más efectivos frente a la delincuencia en los jóvenes (Raya et al., 2009). Junto con estos factores sobresalen otros mecanismos protectores como gozar de una buena situación económica, tener metas elevadas, adaptarse a los roles familiares, participar en la comunidad y en la unidad familiar (Dodge, Bates $\&$ Pettit, 2003).

A continuación, se presenta una revisión teórica de los factores de riesgo sobre los cuales enfatizó esta investigación: Estrés parental y Trato rudo. Posteriormente, se abordará teóricamente el factor protector de Monitoreo o Supervisión.

\section{Factores de riesgo}

\section{Estrés parental}

El estrés de los padres no solo se relaciona con el comportamiento de los hijos, sino con las molestias acumuladas que perciben respecto a su papel. En esta experiencia cotidiana los padres encuentran 
satisfacciones frente a su labor, las cuales generan confianza, pero también, frustraciones y dificultades que los llevan a experimentar estrés.

Lazarus y Folkman (1986) plantean que el estrés se da cuando una situación particular desborda los recursos de la persona afectando su bienestar. Este planteamiento considera como fundamental la relación individuo-entorno, en la cual no siempre es suficiente la sola situación estresante, sino que procesos de afrontamiento inadecuados en el individuo pueden llevar a un resultado no esperado o no deseado, originando una respuesta disfuncional a la exigencia del entorno. Esta definición de estrés también se refiere a un proceso evaluativo que determina hasta qué punto una relación es estresante, y para ello tiene en cuenta factores personales y situacionales.

Siguiendo con Lazarus y Folkman, esta misma situación se puede apreciar en el contexto familiar, en las relaciones padres-hijos, en la angustia o malestar que sienten los padres con las actividades de la crianza, o con el estrés parental. Si no cuentan con los recursos necesarios para enfrentar las demandas de la educación de los hijos, o si el proceso evaluativo de la relación entre padres e hijos es negativo, se origina el estrés parental (Pozo, Sarriá \& Méndez, 2006).

Así, el estudio de Hughes y Huth-Bocks (2007) define el estrés parental como aquellos sentimientos negativos y la angustia que se siente al interior de sí mismo (perspectiva parental) y en lo relacionado con la crianza de los hijos, en el contexto de la parentalidad. El estrés de la crianza se refiere al nivel de competencia de los padres para el cuidado de los hijos y la percepción sobre su temperamento y comportamiento (Burke \& Abidin, 1980; Henao, 2006; Loyd \& Abidin, 1985).

El estrés parental se estudia en los modelos de Abidin y Brunner (1995 citados por Vera \& Peña, 2005) quienes consideran que el estrés está determinado por el apoyo percibido del padre, los recursos y habilidades de la pareja para mantener un estado de equilibrio afectivo y una promoción adecuada del desarrollo del niño. Estos autores concluyen que la percepción de apoyo del padre es una variable importante para explicar el estrés de la crianza de la madre. Es decir, cuando la madre se siente apoyada por el padre en las actividades de la crianza, el nivel de estrés es menor.

La falta de adaptación a la relación de pareja y a la paternidad puede llevar a ambos padres, sobre todo a la madre, a experimentar su maternidad como una actividad estresante, afectando el desarrollo de los hijos. Los eventos de la vida que parecen estresantes tienen efectos sobre la relación funcional padres-hijo (Abidin, 1995; Baker, Blacher \& Olsson, 2005; Karlen, 2004; Webster-Stratton, 1990).

El estrés parental parece tener efectos graves en diferentes áreas del funcionamiento familiar. Se conoce que la percepción de la conducta del niño se modifica por los niveles de estrés de los padres (Webster-Stratton, 1990) y, al mismo tiempo, una percepción distorsionada interviene en la calidad de la interacción madre-hijo. El estrés parental se encuentra asociado con problemas de conducta en los niños y con conductas agresivas de las madres hacia los niños (Herrero, Estévez \& Musitu, 2006; Holden \& Ritchie, 1991; Holden, Stein, Ritchie, Harris \& Jouriles, 1998).

Altos índices de estrés experimentados en las familias se asocian con un número mayor de problemas psicológicos en los hijos (Peligrín, 2004; Peligrín \& Garcés de Los Fayos, 2008; Polansky, Chalmers, Williams \& Buttenwieser, 1981; Sloan \& Meier, 1983; Susman, Tricket, Iannoti, Hollenbeck \& Zahn-Waxler, 1985). En el contexto colombiano, Hernández y Salazar (2003) encontraron que el padre que experimenta estrés parental está en riesgo de abusar de sus hijos siendo indiferente, rechazándolos o abusando físicamente de ellos, esto último desencadenado por la frustración que origina el estrés. Desde este punto de vista, otro factor de riesgo que puede contribuir a la aparición de conductas agresivas en los hijos es el trato rudo.

\section{Trato rudo}

En la investigación de Pettit, Bates y Dodge (1997) se define el trato rudo como una forma de disciplina restrictiva que ejercen padres autoritarios con comportamientos tales como castigo físico frecuente, actitud severa y estricta. Simons, Whitbeck, Conger y Chyi-in (1991) hacen diferencia entre el trato rudo 
y el maltrato o abuso de niños. El abuso combina el daño físico y el rechazo emocional, mientras que el trato rudo no incluye el rechazo emocional del niño.

El trato rudo que ejercen los padres con los hijos se puede reflejar de dos formas: una, consiste en una disciplina agresiva que los padres aplican con el fin de que los hijos hagan lo que ellos desean, sin recurrir a la reflexión y otra, consiste en una serie de pautas normativas incluida la disciplina física estricta que induce a la reflexión. Esto lleva a pensar que el trato rudo se encuentra fundamentado en ciertas creencias de los padres en relación con que esta es la mejor manera de disciplinar a los hijos. Por otra parte, cuando el ajuste psicológico y social de los padres es deficiente, se incrementa el riesgo de que el clima familiar se deteriore y una manifestación potencial de ese deterioro, es el trato rudo.

El estrés de los padres también suele ser asociado con el trato rudo a los hijos. García, Musitu y García (1991) en su investigación con población española tomaron una muestra compuesta por padres y madres que ejercían el trato rudo con sus hijos y otros que no lo ejercían. Los resultados obtenidos permitieron concluir que los padres que ejercen trato rudo hacia sus hijos, experimentan niveles más elevados de estrés.

Por su parte, O’leary y Vidair (2005) examinaron la importancia de dos factores en los problemas de conducta de los niños: los desacuerdos que los padres reflejan en la educación de sus hijos y altos niveles del trato rudo. Encontraron que, completa o parcialmente, estas dos prácticas de los padres median la relación entre el ajuste marital y los problemas de conducta en los niños.

Según Frias-Armenta y McCloskey (1998) existen correlaciones positivas y significativas entre el trato rudo y los problemas de conducta externalizantes en los niños, y asociaciones negativas y significativas con las habilidades sociales y con el desempeño académico.

Por otro lado, Pinderhughes et al. (2000) mencionan que algunos autores han encontrado que el trato rudo contribuye al desarrollo de la conducta antisocial en los niños. El nivel de estrés de los padres está relacionado con el trato rudo que estos ofrecen a sus hijos.

\section{Factor protector}

\section{Monitoreo}

Fletcher, Steinberg y Williams-Wheeler (2004) analizaron la relación entre el consumo de sustancias y el involucramiento en actividades delincuenciales en un grupo de adolescentes, con el monitoreo de los padres de sus actividades diarias. Estos investigadores observaron que el apoyo que se le manifestaba a los hijos, el control y el monitoreo que hacían sus padres de su comportamiento eran los mejores predictores de los problemas de conducta del hijo, pero de manera inversa. Estos investigadores también encontraron que cuando los padres se esforzaban por conocer dónde están sus hijos y qué hacen, estos últimos podían estar menos inclinados a presentar conductas problemáticas.

Fletcher et al. (2004) definieron el monitoreo parental en términos del reporte que hacen los adolescentes sobre la forma como sus padres trataron de obtener información sobre los sitios que frecuentaban, las actividades que hacían y sus amigos más cercanos.

Este estudio asumió el monitoreo como una práctica que ejercen los padres con sus hijos, no de una manera invasiva o intrusiva, sino como producto de una relación que se ha cultivado armoniosamente entre padres e hijos. El hijo siente que debe informar sus actividades a sus padres, porque estos se interesan en él y los padres solicitan la información con el mismo objetivo.

Stattin y Kerr (2000) definen este concepto como el conocimiento de los padres acerca de las actividades de sus hijos fuera del hogar; se da por el esfuerzo de los padres de solicitar y controlar lo que hacen sus hijos y porque estos últimos también comparten voluntariamente información sobre sus actividades.

En esta dirección, Cabrera y Guevara (2007) en su estudio obtuvieron información de padres, madres y adolescentes de la misma familia y mostraron que los padres y madres que reportan altos niveles de monitoreo, aceptación y disciplina inductiva, tienden a apreciar bajos niveles de comportamientos externalizantes en sus hijos adolescentes. Además, 
la versión de los hijos arrojó promedios más altos en la percepción de la mamá en monitoreo, aceptación, disciplina inductiva, control restrictivo y afirmación del poder, a diferencia de la percepción de los hijos sobre el papá.

Como se planteó inicialmente, este estudio se centra en el estrés parental, el trato rudo y el monitoreo asociados a la conducta agresiva de los hijos. Existen investigaciones que han examinado la relación entre el conflicto marital y la aparición de conductas agresivas en los hijos (Ballesteros, 1995; Kerig, Cowan \& Cowan, 1993; O'leary \& Vidair, 2005), pero no se tiene en cuenta la importancia del estrés parental en la aparición de estas conductas. De igual forma, es importante indagar cómo influye el trato rudo en la aparición de la agresión en los hijos, tal como lo han hecho otros estudios (Frias-Armenta \& McCloskey, 1998; Pinderhughes et al., 2000; Pettit et al., 1997), pero para esta investigación se diseñó un modelo que incluye conjuntamente el monitoreo y el estrés parental. El estudio contempló las versiones de padres y madres de una misma pareja en las variables a evaluar, para después obtener un solo indicador en donde se promediaron los dos reportes y así obtener un reporte más confiable.

En este orden de ideas, las preguntas que se formularon en este estudio fueron: iSe relacionan el estrés parental, el trato rudo y el monitoreo al hijo con la presencia de conductas agresivas del hijo?, ¿Existen diferencias significativas entre los diferentes niveles socioeconómicos en función del estrés parental, el trato rudo, el monitoreo y las conductas agresivas de los hijos?, ¿Existen diferencias significativas entre padres y madres en el estrés parental, el trato rudo y el monitoreo al hijo?, ¿Existen diferencias significativas entre padres y madres en su percepción de las conductas agresivas de los hijos?, ¿Qué tanta importancia tienen el estrés parental, el trato rudo y el monitoreo conjuntamente en la explicación de las conductas agresivas los hijos?

\section{Método}

\section{Tipo de investigación}

Este estudio es de tipo descriptivo correlacional.

\section{Participantes}

Se contó con una muestra de 256 parejas conformada por padres (edad entre 28 y $65 ; M=47$ y $D E=7.4$ ) y madres (edad entre 27 y $60 ; M=43$ y $D E=6.70$ ), con hijos adolescentes entre 13 y 18 años de edad. Son personas que residían en la ciudad de Bogotá y cuyo nivel socioeconómico estuvo distribuido así: bajo $32 \%$, medio 35 \% y alto $29 \%$.

\section{Instrumentos}

Se diseñó un cuestionario que recogía información sobre las variables demográficas. El estrés parental se midió con el Índice de Estrés Parental-Forma Corta (PSI/SF) de Abidin (1995). Las preguntas son del estilo de: "Me siento atrapado por mis responsabilidades como padre" y se califican con una escala tipo Likert de 1 (total desacuerdo) a 5 (total acuerdo). Altos puntajes indican alto estrés parental ( $\alpha=0.87$ padres y 0.86 madres). Se aplicó a padres y a madres.

La medición del monitoreo se hizo con la escala de 9 ítems sobre conocimiento de los padres de las conductas de los hijos (Stattin \& Kerr, 2000). Ejemplo: "Mis padres saben quiénes son mis amigos" y la correspondiente versión para padres, en una escala de $1=$ no sabe, a $5=$ sabe mucho $(\alpha=0.91$ para padres, 0.90 madres y 0.88 hijos).

El estudio del trato rudo se llevó a cabo con la escala Harsh Discipline Scale de Straus, Gelles y Steinmetz (1980). Con 8 preguntas como: "cuando su hijo se equivoca, con qué frecuencia le pega o le da palmadas". Se responde en una escala de $1=$ nunca, $5=$ siempre $(\alpha=0.86$ para madres, $\alpha=0.83$ padres y para hijos $\alpha=0.87$ ). A mayor puntaje es mayor el trato rudo ejercido por los padres. De acuerdo con los resultados de la prueba piloto, se diseñaron otros ítems para elevar el índice de confiabilidad del instrumento.

La agresión se evaluó con el Child Behavior Check List (CBCL) de Achenbach y Rescola (2001). Este instrumento evalúa conductas internalizantes y externalizantes y de estas últimas solo se midió la agresión, ejemplo: "se mete mucho en peleas", se 
responde en escala de $0=$ no es cierto a $2=$ muy $a$ menudo ( $\alpha=0.83$ padres, 0.84 madres y 0.81 hijos).

Las preguntas se adaptaron según la persona a quien iban dirigidas, se tradujeron del inglés al español (excepto el de estrés parental), se probó su confiabilidad en el estudio piloto previo y según los resultados se diseñaron nuevas preguntas para elevar el índice de confiabilidad. Los puntajes obtenidos de los informantes (papá, mamá e hijo) se promediaron para obtener un indicador más confiable de cada variable. De esa manera, se simplificó el número de cálculos y se evitó la probabilidad de error en la decisión estadística.

\section{Procedimiento}

Se capacitó a un grupo de estudiantes de Psicología de la Universidad de La Sabana para aplicar los cuestionarios, a manera de entrevista. Se contó con el reporte de los dos padres de las familias del estudio. Su participación fue voluntaria y se logró a través del contacto telefónico o personal. Las entrevistas se realizaron por separado y en momentos diferentes a los dos padres, quienes firmaron el consentimiento informado y registraron sus datos personales.

Los datos fueron sometidos a los siguientes análisis: estadísticos descriptivos de las variables del estudio, correlación entre los puntajes obtenidos en el estrés parental, el trato rudo y el monitoreo. Análisis de una vía ANOVA para las diferencias en los niveles socioeconómicos y las variables del estudio. Prueba $t$ para muestras independientes con el fin de identificar las diferencias entre padres y madres en las variables del estudio y modelo de regresión lineal múltiple para identificar la impor- tancia de las variables del estudio en la explicación de la variabilidad de la conducta agresiva.

\section{Resultados}

\section{Estadísticos descriptivos}

El promedio más alto entre las variables contempladas en este estudio en adolescentes, correspondió al monitoreo, seguido por el estrés de los padres y después el trato rudo al hijo. La agresión obtuvo el promedio más bajo (Tabla 1).

Asociaciones entre el estrés parental, el trato rudo, el monitoreo y las conductas agresivas de los hijos.

El estrés con las actividades de la crianza se asoció directa y significativamente con el trato rudo y las conductas agresivas de los hijos. A su vez, el trato rudo correlacionó de manera significativa y directa con las conductas agresivas de los hijos. Es decir, a mayores niveles de estrés parental, los padres tienden a tratar rudamente a los hijos y estos, a su vez, presentan más conductas agresivas. El trato rudo se asoció significativamente y de manera inversa con el monitoreo, es decir, cuando los padres están pendientes de las actividades de sus hijos tienden a tratarlos con menos rudeza, por el contrario, el trato rudo está directamente asociado con la formación de conductas agresivas en los hijos, o sea, cuando los hijos son agresivos puede asociarse con conductas de trato rudo de sus padres. El monitoreo por su parte, se relaciona de manera inversa y significativa con la aparición de conductas agresivas en los hijos (Tabla 2).

Tabla 1

Promedios y desviaciones estándar de las variables del estudio

\begin{tabular}{lccc}
\hline & Variable & Promedio & DE \\
\hline Estrés de los padres & & 1.99 & 0.86 \\
Trato rudo al hijo & 1.62 & 0.65 \\
Monitoreo al hijo & 4.04 & 0.87 \\
Conductas agresivas del hijo & & 0.47 & 0.34 \\
\hline
\end{tabular}

Fuente: elaboración propia 
TABLA 2

Correlaciones entre el estrés parental, el trato rudo, el monitoreo y las conductas agresivas de los hijos

\begin{tabular}{lcccc}
\hline \multicolumn{1}{c}{ Variable } & 1 & 2 & 3 & 4 \\
\hline 1. Estrés de los padres. & - & $0.27^{* *}$ & $-0.28^{* *}$ & $0.43^{* *}$ \\
2. Trato rudo al hijo. & & - & $-0.24^{* *}$ & $0.42^{* *}$ \\
3. Monitoreo al hijo. & & & - & $-0.3^{* *}$ \\
4. Conductas agresivas de los hijos. & & & & - \\
\hline$* * p<0.01$ & & & \\
Fuente: elaboración propia. & & &
\end{tabular}

Diferencias significativas entre los diferentes niveles socioeconómicos en el estrés parental, el trato rudo, monitoreo y las conductas agresivas

Para analizar las diferencias entre los niveles socioeconómico en el estrés de los padres, el trato rudo, el monitoreo y las conductas agresivas se realizó un ANOVA de una vía.

En cuanto al estrés de los padres, se encontraron diferencias significativas entre los diferentes niveles socioeconómicos $(F(2.461)=6.4 ; p=0.001)$. Igualmente, se encontraron diferencias significativas entre estos niveles según el monitoreo $(F(2.472)$ $=10.2 ; p=0.00)$ y en el trato rudo $(F(2.345)$ $=16.01 ; p=0.00$ ).

Al encontrar diferencias significativas se realizó un análisis post hoc mediante la prueba de Tukey y, en cuanto al estrés de los padres, se encontró que el trato rudo, el monitoreo y las conductas agresivas de los hijos existen diferencias significativas entre los niveles bajo y medio y bajo y alto, pero no entre los niveles medio y alto. En el nivel bajo los padres viven más el estrés parental, tratan con mayor rudeza a los hijos, así como se perciben más conductas agresivas en ellos, en comparación con los niveles medio y alto. Los padres de nivel medio y alto monitorean más a los hijos que los padres de nivel bajo.

Diferencias significativas entre padres y madres en el estrés parental, el trato rudo, el monitoreo y las conductas agresivas de los hijos

Se llevó a cabo una prueba $t$ para muestras independientes. No se encontraron diferencias significativas entre padres y madres en el estrés parental $t(476)=0.46, p=0.65$; y el trato rudo $t(353)=-0.98, p=0.32$. Es decir, tanto los papás como las mamás experimentan estrés con las actividades de la crianza y los dos tratan rudamente a los hijos. Por otro lado, sí se encontraron diferencias significativas entre papás y mamás en el ejercicio del monitoreo a los hijos $t(487)=-4.33, p=0.000$. Es decir, las mamás $(M=4.21)$ presentan niveles más altos de monitoreo de las actividades de los hijos que los papás $(M=3.87)$.

Así mismo, con el fin de identificar si hay diferencias significativas en la versión de padres y madres en su percepción de las conductas agresivas de los hijos, se encontró que no hay diferencias en estas conductas según la percepción de los padres $t(487)=-0.40, p=0.69$. Para los padres, tanto los hijos como las hijas pueden presentar conductas agresivas.

Importancia del estrés parental, el trato rudo y el monitoreo conjuntamente en la explicación de la variabilidad de las conductas agresivas de los hijos.

Se realizó un modelo de regresión lineal múltiple con método introducir para conocer la explicación de la variabilidad de las de conductas agresivas, teniendo como variables independientes el estrés de los padres, el trato rudo y el monitoreo.

El modelo obtenido explica de manera significativa la varianza de la percepción de conductas agresivas. La característica que mayor peso tiene en la explicación es el estrés parental, seguido del trato rudo. El monitoreo también explica significativamente la variabilidad de las conductas agresivas pero de manera inversa, es decir, estar pendiente de las actividades de los hijos previene la aparición de agresión en ellos (Tabla 3). 
TABLA 3

Análisis de regresión para la explicación de la variabilidad de las conductas agresivas de los hijos

\begin{tabular}{lcccc}
\hline & Modelo & $F$ & Beta & $p$ \\
\hline Estrés parental & & 0.34 & 0.000 \\
Trato rudo & & 0.31 & 0.000 \\
Monitoreo & & & -0.10 & 0.04 \\
$R^{2}=0.31$ & $R^{2}$ Ajustado=0.31 & $F(3.313)=47.33 p=0.00$ & & \\
\hline
\end{tabular}

Fuente: elaboración propia.

\section{Discusión}

El propósito de este estudio era indagar si el monitoreo como factor protector y el estrés parental y el trato rudo como factores de riesgo se relacionan y contribuyen en la explicación de la variabilidad de conductas agresivas en los hijos, así como, analizar si existen diferencias significativas entre los diferentes niveles socioeconómicos y entre padres y madres en la vivencia del estrés parental, el trato rudo y el monitoreo. Adicionalmente, se buscó examinar si existen diferencias entre hijos e hijas en la presencia de conductas agresivas según el reporte de los padres. A continuación, se discutirán los principales resultados del estudio a partir de las preguntas de investigación formuladas y de acuerdo con los antecedentes empíricos contemplados en el mismo.

Asociaciones entre el estrés parental, el trato rudo, el monitoreo y las conductas agresivas de los hijos

La conducta agresiva de los hijos se asocia con el estrés parental, el trato rudo y el monitoreo y este resultado del estudio es coincidente con los hallazgos de Abidín (1990) y Pinderhughes et al. (2000). Las tensiones generadas por la crianza se derivan a los demás miembros de la familia, influyendo especialmente en la interacción padres-hijos. Además, la acumulación de eventos estresantes pueden llevar a los padres a ejercer trato rudo hacia sus hijos (Lazarus \& Launier, 1978; Pinderhughes et al., 2000), quienes a su vez responden con conductas externalizantes como la conducta agresiva y la ruptura de normas. Los resultados encontrados coinciden con los de Frias-Armenta y McCloskey (1998), quienes hallaron correlaciones positivas y significativas entre el trato rudo y los problemas de conducta externalizantes en los niños. Igualmente, los resultados encontrados por Hernández y Salazar (2003) con población colombiana plantean que el estrés parental pone en riesgo a los padres para ejercer el trato rudo hacia los hijos.

\section{Diferencias significativas entre los diferentes niveles socioeconómicos en el estrés parental, el trato rudo, el monitoreo y las conductas agresivas}

En consonancia con lo encontrado por Guevara et al. (2007), en este estudio se hallaron diferencias significativas entre los niveles socioeconómicos bajo y medio y bajo y alto en términos del monitoreo de los padres y las conductas agresivas de los hijos. Adicionalmente, se encontró asociación entre el nivel socioeconómico bajo y las prácticas de rudeza y estrés parental, mientras que los niveles medio y alto se encontraron mayores niveles de monitoreo. En este sentido, es posible que en los niveles bajos se presenten mayores conductas agresivas de los hijos que en los niveles medio y alto (Belsky, 1984; Peligrín \& Garcés de los Fayos, 2008). Así, este resultado señala la importancia de girar la atención a los niveles socioeconómicos más bajos, para generar planes de acción que contemplen factores protectores y reduzcan los factores de riesgo y así contribuyan a la prevención de conductas agresivas de los hijos.

\section{Diferencias significativas entre padres y madres en el estrés parental, el trato rudo, el monitoreo y las conductas agresivas de los hijos}

El factor protector (monitoreo) presentó diferencias significativas en los reportes de padres y madres, 
a diferencia de los factores de riesgo, trato rudo y estrés parental en los cuales no se evidenciaron diferencias. Este resultado coincide con lo hallado por Cabrera y Guevara (2007) en su estudio. Desde este punto de vista, el monitoreo es un factor protector frente a la conducta agresiva tal como lo plantean Fletcher et al. (2004), al romper la dinámica con los factores de estrés parental y trato rudo, ya que el monitoreo puede dar a los padres una percepción de confianza sobre la crianza de sus hijos, evitando el estrés y a su vez disminuyendo las posibilidades de trato rudo.

No se encontraron diferencias significativas entre las percepciones de padres y madres sobre la conducta agresiva de los hijos, es decir, tanto los hombres como las mujeres pueden presentar estas conductas. En contraste con lo encontrado en otros estudios, los hombres tienden a presentar más conductas agresivas que las mujeres (Tremblay et al., 2008; Webster-Stratton, 2003).

\section{Importancia del estrés parental, el trato rudo y el monitoreo en la explicación de la variabilidad de las conductas agresivas de los hijos}

El modelo planteado para explicar las conductas agresivas de los hijos según el reporte de padres y madres fue significativo. Aunque los tres factores se asocian con la conducta agresiva de los hijos, el que mayor peso tiene es el estrés parental que puede desencadenar trato rudo y por ende, la conducta agresiva. Así lo señalan Ayala et al. (2002) quienes encontraron que cuando el padre o la madre se sienten estresados para asumir su rol parental, se pueden desencadenar conductas agresivas en los hijos.

El modelo propuesto para esta investigación (estrés parental, trato rudo y monitoreo) es relevante en al considerar que en estudios previos estos factores han sido analizados de forma separada o en relación con otros factores (Ayala et al., 2002; Dishion \& McMahon; 1998; Kazdin, 1993; Patterson, Reid \& Dishion, 1992), pero no en un modelo conjunto que integre las tres practicas parentales ya descritas e identificadas en la literatura como aquellas que con mayor frecuencia se asocian a la conducta agresiva de los hijos. En análisis conjunto permite no solamente ver cuál tiene mayor relevancia en la explicación de la variabilidad de la conducta agresiva, sino también identificar una dinámica en la que los factores se retroalimentan para generar o prevenir la aparición de la conducta externalizante.

Por otra parte, el monitoreo contribuye como factor protector a la aparición de la conducta agresiva, es decir, los padres que monitorean a los hijos disminuyen la probabilidad de formación de conductas agresivas en ellos, lo que apoya lo reportado por Werner (1986), Luthar (1993) y Dunst y Trivett (1994).

Entonces, así como existen factores familiares que desencadenan la agresión en los hijos también se hallan otros que protegen la formación de esta conducta poco deseable para la sociedad, como estar al tanto de las actividades de los hijos. Se puede concluir que tanto los factores protectores, en este caso el monitoreo, como los factores de riesgo, contribuyen a que los hijos desarrollen o no conductas agresivas, los primeros de manera inversa y los segundos de manera directa.

Para futuros desarrollos en investigación, se pueden adelantar estudios que evalúen las conductas agresivas en familias monoparentales y hacer comparaciones entre familias con madre cabeza de hogar y padre cabeza de hogar. Como lo sugieren Achenbach y Rescola (2001), se pueden desarrollar otros estudios a partir de los reportes de los profesores acerca del comportamiento agresivo de los adolescentes y de los reportes de los mismos adolescentes con el fin conocer sus percepciones.

De acuerdo con los resultados obtenidos, se pueden generar programas que capaciten a los padres para favorecer el ambiente familiar sano, a través de la utilización de prácticas parentales que funcionen como factores protectores, en este caso el monitoreo y busquen prevenir la formación de factores de riesgo como el estrés relacionado con el ejercicio de la maternidad y la paternidad, el trato rudo de los hijos u otros factores como el retiro del afecto o el conflicto marital. 


\section{Referencias}

Abidin, R. (1995). Parenting stress index: Professional manual ( $3^{\text {a }}$ ed.). Odessa, Fl: Psychological Assessment Resources.

Abidin, R. \& Brunner, J. (1995). Development of a parenting alliance inventory. Journal of Clinical Child Psychology, 24, 31-40.

Achenbach, T. \& Rescorla, L. (2001). Manual for the ASEBA school-age forms and profiles. Child behavior checklist for age 6-18, teacher's report from, youth self-report and integrated system of multi-informant assessment. Burlington, VT: University of Vermont.

Ayala, H., Fulgencio, M., Chaparro, A. \& Pedroza, F. (2000). Resultados preliminares del proyecto estudio longitudinal del desarrollo de la conducta agresiva en niños y su relación con el establecimiento de conducta antisocial en la adolescencia. Revista Mexicana Análisis de la Conducta, 26, 65-89.

Ayala, H., Pedroza, F., Morales, S., Chaparro, A. \& Barragán, N. (2002). Factores de riesgo, factores protectores y generalización del comportamiento agresivo en una muestra de niños en edad escolar. Salud Mental, 25(3), 27-40.

Ballesteros de Valderrama, B. (1995). El conflicto marital y su relación con problemas de ajuste en los niños. Trabajo monográfico, Fundación Universitaria Konrad Lorenz, Bogotá, Colombia.

Baker, B., Blacher, J. \& Olsson, M. (2005). Preschool children with and without developmental delay: Behaviour problems, parents' optimism and wellbeing. Journal of American Child and Adolescent Psychiatric, 44(3), 575-590.

Barkley, R. (1997). Defiant children: A clinician's manual for assessment and parent training. Nueva York: The Guilford Press.

Belsky, J. (1984). The determinants of parenting: Process model. Child Development, 55, 83-96.

Björkvist, K. (2001). Top ten challenges for understanding gender and aggression in children: Why can't we all just get along? Social Development, 10(2), 248-266.

Burke, W. \& Abidin, R. (1980). Parenting stress index (PSI): A family system assessment approach. En R. R. Abidin (Ed.), Parent education and intervention handbook (pp. 516-527). Spriengfield, IL: Thomas.
Cabrera, V. \& Guevara, I. (2007). Relaciones familiares y ajuste psicológico: dos estudios en adolescentes de familias colombianas. Bogotá: Ediciones Uniandes.

Cavell, T. (2000). Working with parents of aggressive children. A practitioner's guide. Washington: American Psychological Association.

Chaux, E. (2003). Agresión reactiva, agresión proactiva y el ciclo de la violencia. Revista de Estudios Sociales, 15, 47-58.

Côté, S. M., Vaillancourt, T., Barker, E. D., Nagin, D. S. \& Tremblay, R. E. (2007). The joint development of physical and indirect aggression: Predictors of continuity and change during childhood. Development and Psychopathology, 19, 37-55.

Crick, N., Casas, J. \& Mosher, M. (1997). Relational and overt aggression in preschool. Developmental Psychology, 33(4), 579-588.

Crick, N. \& Pepler, D. (2007). Understanding bullying: From research to practice. Canadian Psychology, 48(2), 86-93.

Dekovic, M., Wissink, I. \& Meijer, A. (2004). The role of family and peer relations in adolescent antisocial behaviour: Comparison of four ethnic groups. Journal of Adolescence, 27, 497-514.

Dishion, T. J. \& McMahon, R. J. (1998). Parental monitoring and the prevention of child and adolescent problem behavior: A conceptual and empirical formulation. Clinical Child and Family Psychology Review, 1, 61-75.

Dodge, K., Bates, J. \& Pettit, G. (2003). Preventing aggressive behavior early in life: Comments on Webster-Stratton, Lochman, and Domitrovich and Greenberg. En R. E. Tremblay, R. G. Barr \& R. de V. Peters (Eds.), Encyclopedia on Early Childhood Development (pp. 1-4). Montreal, Quebec: Centre of Excellence for early Childhood Development.

Dodge, K., Pettit, G., McClaskey, C. \& Brown, M. (1986). Social competence in children. Monographs of the Society for Research in Child Development, 51, 1-85.

Dunst, C. \& Trivette, C. (1994). Methodological considerations and strategies for studying the long-term followup of early interventions. En S. Friedman $\&$ H. Haywood (Eds), Developmental follow-up: Concepts, domains and methods (pp. 277-313). San Diego: Academic Press. 
Farrington, D. (1989). Early predictors of adolescent aggression and adult violence. Violens and Victims, 4, 79-100.

Fletcher, A., Steinberg, L. \& Williams-Wheeler, M. (2004). Parental influences on adolescent problem behavior: Revisiting Sttatin and Kerr. Child Development, 75(3), 781-796.

Frias-Armenta, M. \& McCloskey, L. (1998). Determinants of harsh parenting. Journal of Abnormal Child Psychology, 26(2), 129-139.

Galen, B. \& Underwood, M. (1997). A developmental investigation of social aggression among children. Developmental Psychology, 4, 589-600.

García, E., Musito, G. \& García, F. (1991). Sucesos y cambios vitales estresantes y ajuste psicológico: un análisis del apoyo social como variable moduladora en padres normales y padres que maltratan a sus hijos. Iniciativas, 3, 81-94.

Gladstone, G., Parker, G. \& Malhi, G. (2006). Do bullied children become anxious and depressed adults? A cross-sectional investigation of the correlates of bullying and anxious depression. The Journal of Nervous and Mental Disease, 194(3), 201-208.

Guevara, I., Cabrera, V. \& Barrera, F. (2007). Factores contextuales y emociones morales como predictores del ajuste psicológico en la adolescencia. Revista Universitas Psychologica, 6(2), 269-283.

Henao, J. (2006). La prevención temprana de la violencia: una revisión de programas y modalidades de intervención. Infancia, Adolescencia y Familia, 1(2), 315-339.

Hernández, D. \& Salazar, A. (2003). Estrés parental y laboral, conflicto trabajo-familia y ansiedad en hombres. Bogotá: Ediciones Uniandes.

Herrero, J., Estévez, E., \& Musitu, G. (2006). The Relationships of adolescent school-related deviant behaviour and victimization with psychological distress: Testing a general model of the mediational role of parents and teachers across groups of gender and age. Journal of Adolescence, 29(5), 671-690.

Holden, G. \& Ritchie, K. (1991). Linking extreme marital discord, child rearing, and child behavior problems: Evidence from battered women. Child Development, 62, 311-327.

Holden, G., Stein, J., Ritchie, K., Harris, S. \& Jouriles, E. (1998). Parenting behavior and beliefs of bat- tered women. En G. W. Holden, R. Geffner \& E. N. Jouriles (Eds.), Children exposed to marital violence. Theory, research and applied issues (pp. 289. 234). Washington D.C.: American Psychological Association.

Hughes, H. M. \& Huth-Bocks, A. C. (2007). Variations in parenting stress in African-American battered women: Implications for children's adjustment and family intervention. European Psychologist, 12(1), 62-71.

Karlen, R. (2004). La disociación y el diálogo infantoparental: una perspectiva longitudinal a partir de la investigación sobre apego. Revista Internacional de Psicoanálisis, 17. Disponible en http://www.aperturas.org/terminos.php? $\mathrm{t}=$ neu

Kazdin, A. (1993). Adolescent mental health: Prevention and treatment programs. American Psychologist, 48, 127-141.

Kerig, P., Cowan, P. \& Cowan, C. (1993). Marital quality and gender differences in parent-child interaction. Development Psychology, 29(6), 931-939.

Kumpfer, K. L. \& Alvarado, R. (1997). Effective Family Strengthening Interventions [Bulletin]. Washington, DC: U.S. Department of Justice, Office of Justice Programs, Office of Juvenile Justice and Delinquency Prevention.

Lazarus, R. \& Folkman, S. (1986). Estrés y procesos cognitivos. Barcelona: Martínez Roca.

Lazarus, R. \& Laurnier, R. (1978). Stress related transactions between person and environment. En L. A. Pervin \& M. Lewis (Eds.), Perspectives in interactional psychology (pp. 287-327). New York: Plenum.

Loyd, B. \& Abidin, R. (1985). Revision of the Parenting Stress Index. Journal of Pediatric Psychology, 10(2), 169-177.

Luthars, S. (1993). Methodological and conceptual issues in research on childhood resilience. Journal of Child Psychology and Psychiatry, 34(4), 441-453.

Morales, M., García de la Cadena, C. \& Grazioso, M. (2006). Conciencia sobre intimidación en la formación de maestras de párvulos. Revista Interamericana de Psicología, 1, 69-76.

O'leary, S. \& Vidair, H. (2005). Marital adjustment, child-rearing disagreements, and overreactive parenting: Predicting child behavior problems. Journal of Family Psychology, 19(2), 208-216. 
Olweus, D. (1993). Conductas de acoso y amenaza entre escolares. Madrid: Morata.

Patterson, G., Reid, J. \& Dishion, T. (1992). Antisocial Boys. Eugene, OR: Castalia Publishing Company.

Peligrín, A. (2004). El comportamiento agresivo y violento: factores de riesgo y protección como mediadores de inadaptaciones y adaptaciones en la socialización del niño y el adolescente. Tesis doctoral no publicada, Universidad de Murcia, Murcia, España.

Peligrín, A. \& Garcés, E. (2008). Variables contextuales y personales que inciden en el comportamiento violento del niño. European Journal of Education and Psychology, 1, 5-20.

Pettit, G., Bates, J. \& Dodge, K. (1997). Supportive parenting, ecological context, and children's adjustment: A seven-year longitudinal study. Child Development, 68(5), 908-923.

Pinderhughes, E., Dodge, K., Bates, J., Pettit, G. \& Zelli, A. (2000). Discipline responses influences of parents' socioeconomic status, ethnicity, beliefs about parenting, stress and cognitive-emotional processes. Journal of Family Psychology, 14(3), 380-400.

Polansky, N., Chalmers, M., Williams, D. \& Buttenwieser, E. (1981). Damaged parents: An anatomy of child neglect. Chicago, IL: University of Chicago Press.

Pozo, P., Sarriá, E. \& Méndez, L. (2006). Estrés en madres de personas con trastornos del espectro autista. Psicothema, 18, 342-347.

Raya, A., Pino, J. \& Herruzo, J. (2009). La agresividad en la infancia: el estilo de crianza parental como factor relacionado. European Journal of Education and Psychology, 2(3), 211-222.

Richaud de Minzi, M. (2002). Inventario acerca de la percepción que tienen los niños y las niñas de las relaciones con sus padres y madres. Versión para 4 a 6 años. Revista Interamericana de Psicología, 36(1-2), 149-165.

Salmivalli, C. \& Kaukiainen, A. (2004). Female aggression revisited: Variable-and person-centered approaches to studying gender differences in different types of aggression. Aggressive Behavior, 30(2), 158-163.

Salmivalli, C., Lagerspetz, K., Björkqvist, K., Österman, K. \& Kaukiainen, A. (1996). Bullying as a group process: Participant roles and their relations to social status within the group. Aggressive Behavior, 22, 1-15.

Simons, R., Whitbeck, L., Conger, R. \& Chyi-in, W. (1991). Intergenerational transmission of harsh parenting. Developmental Psychology, 27(1), 159 . 171.

Sloan, M. \& Meier, J. (1983). Typology for parents of abused children. Child Abuse and Neglect, 7(4), 443-450.

Stattin, H. \& Kerr, M. (2000). Parental monitoring: A reinterpretation. Child Development, 71, 1072-1085.

Stone, G., Buehler, C. \& Barber, B. (2002). Interparental conflict, parental psychological control and youth problem behavior. En B. K. Barber (Ed.), Intrusive parenting: How psychological control affects children and adolescents (pp. 53-95). Washington, DC: American Psychological Association.

Straus, M., Gelles, R. \& Steinmetz, S. (1980). Behind closed doors: Violence in the American family. Beverly Hills, CA: Sage.

Susman, E., Tricket, P., Iannoti, R., Hollenbeck, B. \& Zahn-Waxler, C. (1985). Child rearing pattems in depressed, abusive and normal mothers. American Journal of Orthopsychiatry, 55(2), 237-251.

Tremblay, R., Gervais, J. \& Petitclerc, A., (2008). Early childhood learning prevents youth violence. Montreal, Quebec: Centre of Excellence for early Childhood Development, CEECD.

Urra, J. (1998). Violencia y medios de comunicación. En J. Sanmartín, J. Grisolia \& S. Grisolia (Eds.), Violencia, televisión y cine (pp. 133-145). Barcelona: Ariel.

Vera, J. \& Peña, M. (2005). Desarrollo, estimulación y estrés de la crianza en infantes rurales de México. Apuntes de Psicología, 23(3), 305-319.

Wahler, R. (1980). The insular mother: Her problems in parent-child treatment. Journal of Applied Behavior Analysis, 13(2), 207-219.

Webster-Stratton, C. (1990). Stress: A potential disruptor of parent perceptions and family interactions. Journal of Clinical Child Psychopatology, 19, 302-312.

Webster-Stratton, C. (2003). Aggression in Young Children Services Proven to be Effective in Reducing Aggression. En R. E. Tremblay, R. G. Barr \& R. de V. Peters (Eds.), Encyclopedia on Early Childhood Development Montreal (pp. 1-6). Montreal, 
Quebec: Centre of Excellence for early Childhood Development.

Werner, E. (1986). Resilient offspring of alcoholics: A longitudinal study from birth to age 18. Journal of Studies on Alcohol and Drugs, 47(1), 34-40. 